confirmation; syphilis using TPPA/VDRL confirmation for active syphilis.

Results A total of 316 female adolescents and 384 males participated. A history of sexual activity was reported by $76.0 \%$ of females and $78.3 \%$ of males; forced sexual intercourse by $29.0 \%(86 / 297)$ of females and $15.1 \%(55 / 364)$ of males; transactional sex (being offered money, food, housing, transport in exchange for sex) by $14.6 \%(43 / 295)$ of females, $12.3 \%(45 /$ $365)$ of males. Of sexually experienced participants, 4.7\%(25/ 535 ) had a confirmed HIV (1 female, 3 males) or active syphilis (3 females, 19 males) test. Correlates of either HIV/syphilis infection include: male gender $(\mathrm{AOR}=4.4,95 \% \mathrm{CI}$ : 1.5-13.1) and having been offered transactional sex $(\mathrm{AOR}=3.3,95 \% \mathrm{CI}$ : 1.3-8.0). Among males only, 4/26 (15.4\%) of those who reported same-sex sexual intercourse (MSM) tested positive for syphilis. Correlates of HIV/syphilis among all male participants included transactional sex $(\mathrm{AOR}=2.7,95 \% \mathrm{CI}: 1.0-7.9)$, having been forced to have sex $(\mathrm{OR}=2.6,95 \% \mathrm{CI}$ : 1.0-6.9) and MSM (OR=2.7, 95\%CI: 0.9-8.9).

Conclusion There is very high syphilis and HIV prevalence among high-school-going indigenous adolescents of $\mathrm{CNB}$, particularly among males. Transactional sex and forced sex are driving factors. Targeted interventions should include prevention education, testing and treatment, especially for boys reporting homosexual sex.

Disclosure No significant relationships.

\section{P580 PREDICTORS OF SEXUALLY TRANSMITTED INFECTION SCREENING AMONG BINGE ALCOHOL USING NATIVE AMERICAN ADULTS}

${ }^{1}$ Rachel Chambers, ${ }^{2}$ Lauren Tingey, ${ }^{2}$ Shea Littlepage, 'Anna Beach, 'Laura Melgar, ${ }^{1}$ Angie Lee, 'Anne Rompalo. 'Johns Hopkins Center for American Indian Health, Baltimore, USA; ${ }^{2}$ Johns Hopkins School of Public Health, Baltimore, USA

\subsection{6/sextrans-2019-sti.653}

Background: Introduction Native American (Native) adults have higher rates of sexually transmitted infections (STIs) than other racial/ethnic groups in the United States. The Centers for Disease Control and Prevention (CDC) recommend annual STI screening for those with new or multiple sex partners. Scarce research has been conducted exploring what factors predict STI screening among high risk Native adults and the extent of compliance with CDC recommendations in this population. This analysis bridges this gap in the literature.

Methods Participants are $\mathrm{N}=252$ Native adults ages 18 to 55 who recently engaged in binge alcohol use and enrolled in a trial evaluating a risk-reduction counseling program. Data were collected at baseline via self-report and assessed participant demographics, substance use, sexual risk behaviors and mental health symptoms. Bivariate analyses followed by final multivariate regression models explored which factors significantly predicted STI screening behavior in the 12 months prior to assessment.

Results Less than half of all participants completed STI screening in the past year $(n=96,38.1 \%)$. Among females, using a condom at last sex and having sex with more than one person in the same day was associated with lower odds of STI testing $(p=0.03, p=0.02)$ while having sex with someone with an STI was associated with higher odds of testing $(p=0.03)$. Among males, drug use and having more than 5 drinks in the same day were associated with higher odds of STI testing $(\mathrm{p}=0.008, \mathrm{p}=0.031)$.

Conclusion Regular STI screening is a key prevention strategy for reducing transmission of STIs, particularly among high risk Native American adults. Understanding what factors predict compliance with CDC recommendations can help inform programming development for Native adults who engage in binge alcohol use. Results inform intervention efforts for CDC, Indian Health Service and other tribal-serving organizations working to increase STI screening rates in Native communities.

Disclosure No significant relationships.

\section{P583 THE ROLE OF PARENTS IN PREDICTING SEXUAL HEALTH AND SUBSTANCE USE RISK AMONG NATIVE AMERICAN YOUTH}

${ }^{1}$ Lauren Tingey*, ${ }^{2}$ Rachel Chambers, ${ }^{3}$ Shea Littlepage, ${ }^{2}$ Anna Beach, ${ }^{2}$ Laura Melgar, ${ }^{2}$ Angie Lee, ${ }^{2}$ Anne Rompalo. 'Johns Hopkins University, Johns Hopkins Center for American Indian Health, Baltimore, USA; ${ }^{2} J o h n s$ Hopkins Center for American Indian Health, Baltimore, USA; ${ }^{3}$ Johns Hopkins School of Public Health, Baltimore, USA

\subsection{6/sextrans-2019-sti.654}

Background Native American youth suffer marked disparities in sexual health and substance use. High rates of sexually transmitted infections (STIs), unintended pregnancies and substance abuse are driven by early sexual and substance use initiation and limited access to culturally relevant health education. Parents and extended family members are central to youth decision making in Native communities. Despite this inherent strength, few health education programs are designed to be multigenerational and fail to engage parents and family members in intervention delivery.

Methods Data was collected with $\mathrm{N}=536$ Native youth ages 11-19 participating in an evaluation of a comprehensive sexual health program designed for youth together with their parents or other trusted adult family members, called Respecting the Circle of Life. Baseline data was analyzed to determine how parental monitoring and parent-youth communication predicted sexual and substance use risk prior to intervention implementation. Bivariate and multivariate logistic regression models examined the role of parents in predicting substance use initiation, sexual initiation (vaginal/anal/ oral), condom use at last sex, as well as intention to have sex and condom use intention.

Results Final multivariate models indicate parental monitoring significantly predicted: initiation of cigarettes $(p=0.02)$, initiation of marijuana $(p=0.001)$, initiation of vaginal sex $(p=0.03)$, initiation of oral sex $(p=0.04)$, intention to have sex in the next 6 months $(p=0.000)$ and intention to use a condom in the next 6 months $(p=0.002)$.

Conclusion Our findings underscore the powerful role of parents and family in Native American sexual and substance use decision making. Results show Native families can help delay youth's initiation of sex and substances and protect them from the consequences of sexual risk taking. Programs aiming to promote the sexual health of Native youth should incorporate parents and other families in lesson delivery and skill development to maximize impact.

Disclosure No significant relationships. 